\title{
Effect of obesity on several types of cancer
}

\author{
Yushi Zhao ${ }^{1, *}$, a, $\dagger$, Tianyi Yang ${ }^{2, *}, \mathrm{~b}, \dagger$ \\ ${ }^{1}$ Fuyuan British American School, 518101 Shenzhen, China \\ ${ }^{2}$ Shanghai DTD Academy, 201105 Shanghai, China \\ ${ }^{\dagger}$ These authors contributed equally.
}

\begin{abstract}
Currently, both trends of obesity and awareness of the possible adverse effects of obesity are rising in our population. According to the World Health Organization, about one third of the world's population is suffering from obesity-related health problems. Cancer, a severe progressive disease that is becoming more and more common, is among them. However, the association between obesity and cancer is not informed very well by the public. In this paper, the first part will briefly introduce present situations of obesity and common complications of obesity. The following two parts will explain the mechanisms of cancer formation and discuss the relationship between obesity and cancer in an understandable way.
\end{abstract}

\section{INTRODUCTION}

The proportion of obesity worldwide is increasing every year. More than 2 billion people are overweight or obese, which means that about one third of the world's population is suffering from overweight or obesityrelated health problems. The rate of increase in the proportion of the obese is increasing much faster. In this situation, obesity also causes various problems. One of its major impacts, is that obesity may cause cancer in the body. Evidence on the relation between obesity and cancer is counting out. Obesity has now been analyzed to cause 13 types of cancers in different parts of the body.

The reason for writing this review is that we have a deeper basis for research on the relationship between obesity and cancer, so we can get more information from those studies. So we can discuss the inflammation and cancer caused by obesity; and the relationship between the change in hormone and cancer.

In this review, we will talk about obesity and what obesity would cause to the patient and the further relationship with cancer such as liver cancer.

\subsection{The current situation and impact of obesity}

\subsubsection{The current situation and impact of obesity}

Worldwide, the standard for obesity is using the value of BMI of a person compared to the comparative table to find out what section that the person belonged.

Normally, the people who have BMI lower than 18.5 is classified as 'underweight', the value of BMI between 18.5 and 25 are classified as 'normal', the value of BMI which is greater than 25 are classified as 'overweight', and those who has BMI greater than 30 are classified as 'obese'.
In this review, the judging standard for obesity is $\mathrm{BMI} \geq 30$. For different countries, the obesity rate is all different. So, we use the standard of WTO, the people with a $B M I \geq 0$ of 30 are classified as obese. More than 2 billion people are overweight or obese, which means that about one third of the world's population is suffering from overweight or obesity-related health problems. Among those obese people globally, 108 million are children, and 600 million are adults, which means 5\% of children and $12 \%$ of adults are obese. Although it shows that the percentage of children is still low, the proportion of obese children increases at a high rate.

The overweight + obese percentages for the overall US population are higher reaching $39.4 \%$ in 1997 , $44.5 \%$ in $2004,56.6 \%$ in $2007,63.8 \%$ (adults) and $17 \%$ (children) in 2008, in 2010. 65.7\% of American adults and $17 \%$ of American children are overweight or obese, and $63 \%$ of teenage girls become overweight by age 11 . In 2013 the Organization for Economic Co-operation and Development (OECD) found that $57.6 \%$ of all American citizens were overweight or obese.

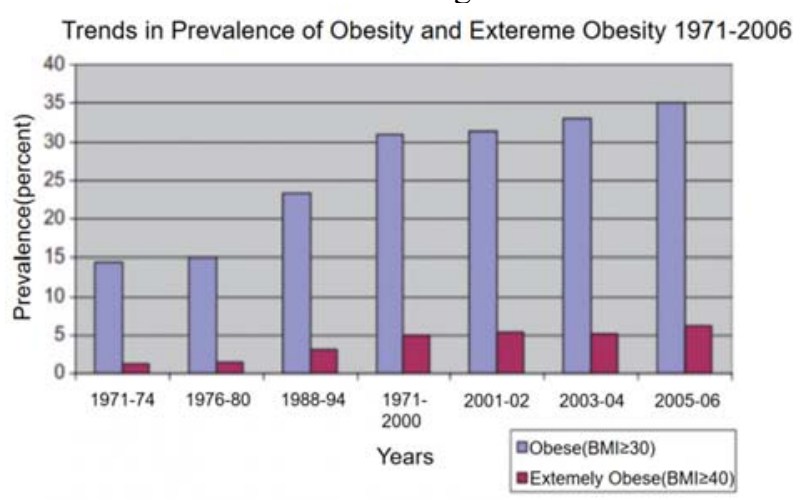

Figure 1 Trends in obesity, U.S. [1] Abbreviation: BMI, body mass index.

\footnotetext{
* Corresponding author:a1254952543@qq.com, byangty@student.dtd-edu.cn.
} 


\subsection{Effects of obesity}

The patient with obesity may have some risk factors of arteriosclerosis like high blood pressure, high blood sugar, high blood lipid, etc. Those factors may increase the risk ratio of Cerebrovascular Diseases (CVD).

\subsubsection{Impact of obesity on the heart}

There are lots of impacts of obesity on the heart. Because obese patients tend to be accompanied by various metabolic diseases, they will be accompanied by high blood pressure, diabetes, blood lipid abnormalities. Especially obese patients are often accompanied by snoring which is called Obstructive Sleep Apnea Syndrome (OSAS). That's due to the fats accumulate around the heart. The heart is unable to beat efficiently and needs to use lots of energy. That will cause the flow of blood to slower. Lipids may attach to the surface of blood vessels. Obese people have thicker subcutaneous fat, which will greatly expand capillaries, increase blood volume. Blood circulation is also relatively increased, thus increasing the load of blood capacity. At normal heart rate, the amount of cardiac arrest increases significantly. The burden on the heart and blood vessels is too heavy for a long time, which can induce left heart hypertrophy and lead to higher blood pressure.

After those risk factors multiply occurs, patients are very likely to combine coronary heart disease. Atherosclerosis may happen. After the combination of coronary heart disease, patients can appear angina, in severe cases can appear myocardial infarction. In addition, obese patients tend to have a large heart and a significant process of ventricular remodeling. And after the enlarged heart affects the function of the heart diastole, the heart's compliance will be significantly affected.

So, when several factors are superimposed, obese patients have coronary heart disease, and the risk of heart failure increases significantly. In particular, obese patients are often accompanied by nighttime OSAS. Nocturnal airway resistance significantly increased, will increase the load before and after the heart, coronary heart disease risk will be further increased.

\subsubsection{Obesity will cause diabetes}

When a person is in the situation of obesity, there will be lots of cholesterol and fats accumulate on the tunica intima, reducing spaces for the transport of blood and will block up lots of capillaries and reduce the consumption rate of sugar. This will cause the ratio of sugar to insulin to increase. The efficiency of insulin is less significant, therefore will cause high blood sugar level and getting diabetes for a long period under this situation. Diabetes has various phenomenons. Like fatty liver, they are having gout, high blood sugar level, Osteoporosis, and much easier to aggravate the infection of pathogens.

\subsubsection{Impact of obesity on the cancer}

Although evidence shows that adult overweight and obesity are related to the risk for many cancers, the growing epidemic of obesity provides a challenge to clinical practice and the implementation of guidelines for weight management. Historical data from the past 25 years point to obesity as a cause of approximately $14 \%$ of cancer deaths in men and up to $20 \%$ of cancer deaths in women [2].

Worldwide, colorectal cancer is the third 1 most common cancer in men and the second2 leading malignancy in women, accounting for 10.0 and $9.2 \%$ of all cancer cases, respectively [3]. In 2012, approximately 746,000 new colorectal cancer cases were diagnosed in men, and 614,000 were diagnosed in women [3]. Agestandardized incidence rates (ASRs) vary widely across the world. Almost $55 \%$ of all cases occur in economically more developed regions such as Australia/New Zealand (men: 44.8 per 100,000, women: 32.2), Europe (men: 37.3, women: 23.6) and Northern America (men: 30.1, women: 22.7) [3]. Incidence rates are lowest in economically less developed regions such as Africa (men: 7.0, women: 5.8) and South Central Asia (men: 7.0, women: 5.2) [3]. Colorectal cancer is the fourth 3 most common cause of death from cancer, contributing to $8.5 \%$ of total cancer mortality worldwide (694,000 deaths per year in both sexes) [3].

Far fewer studies address weight or weight change and survival following a cancer diagnosis. A longstanding association between obesity and poor outcomes for women with breast cancer $[4,5]$ has received increasing attention [6]. The majority of evidence points to weight at diagnosis as the major lifestyle risk for poor breast cancer outcomes (and poorer quality of life) [7], with growing evidence that weight gain after diagnosis exacerbates risk [6] - a result seen most clearly among women who were lean at diagnosis or nonsmokers. Nonsmoking women who gained greater than $2 \mathrm{~kg} / \mathrm{m} 2$ after an abreast cancer diagnosis had a relative risk for death during 9 years of follow-up that was $1.64(95 \% \mathrm{CI}$, 1.07-2.51), compared with stable weight women (Fig 2). Insulin pathways have been suggested as one mechanism for this effect [8].

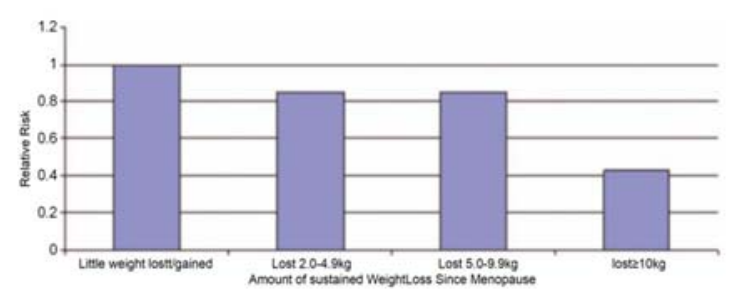

Figure 2 Sustained weight loss and breast cancer risk in postmenopausal women who never used postmenopausal hormones.

Based on Table 3 of Eliassen AH, Colditz G, Rosner B et al. Adult weight change and risk of postmenopausal breast cancer. 


\section{Cancer formation on human body and inducers for cancer}

\subsection{Cancer formation}

\subsubsection{Process of gene mutations causing cancer}

Cancer is the name given to a series of diseases caused by abnormal cells. These cells, after mutations, possess the ability to divide uncontrollably and become able to invade other parts of the body. Commonly, normal cells will undergo a process called a cell cycle, which involves cell growth, cell division, and finally, apoptosis triggered cell death. Any mutations on cell genetic materials that interfere with the normal cycle can cause cancer. For example, the p53 gene is a critical gene that functions in tumor suppressing. Once DNA is damaged, p53 will become active and acts as a transcription factor of a protein that inhibits the cell cycle (see Figure 2). Defective p53 will cause increased cell division. Take Ras protein as another example. It behaves as a $G$ protein that relays a signal from a growth factor receptor to a protein kinase cascade. A mutation in Ras protein will overexpress a protein that stimulates the cell cycle and thus levels the rate of cell division [9].

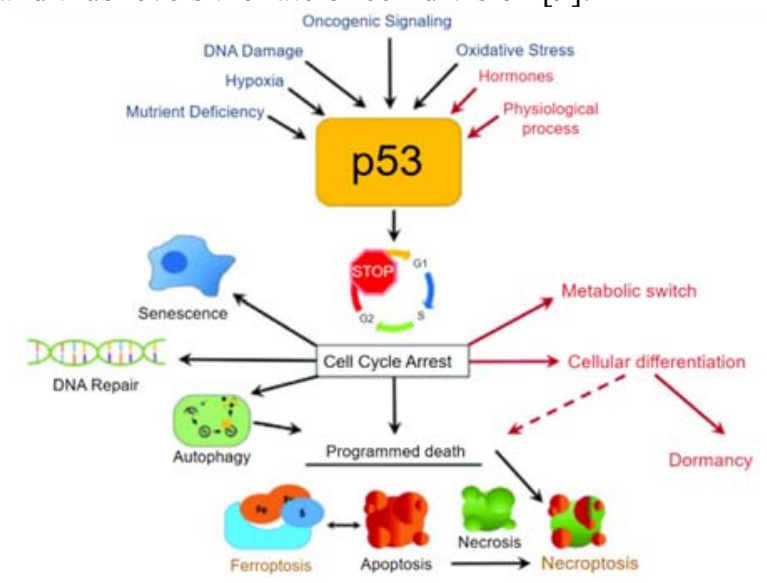

Figure 3 Function of p53 gene

\subsubsection{Stages of cancer on microscopic view (tumor)}

Tumor development begins when some cell within a normal population obtains a genetic mutation that increases its propensity to proliferate. The altered cell will uncontrollably overproduce. And the collection of these cells will enter a stage called hyperplasia. At this stage, instead of being normally flat, the surface will look prominent. Further mutations that deviate from the normal cell cycle make offspring of this cell appear abnormal in shape and orientation; the tissue is now said to exhibit dysplasia. If progressing genetic changes allow the tumor to begin invading underlying tissue and to shed cells into the blood or lymph, the mass is considered to have become malignant [10].
Normal Cells May Become Cancer Cells

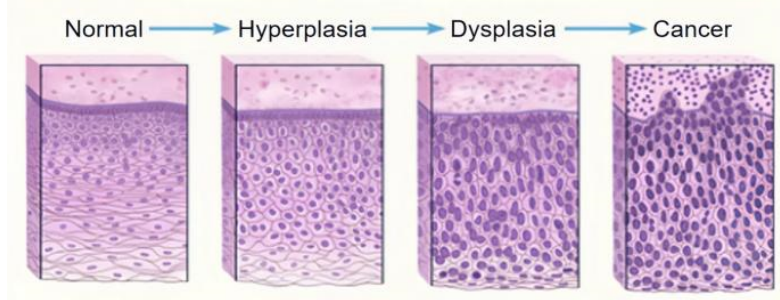

Figure 4 Process of tumor developing

\subsubsection{Cancer expression on the macroscopic view (body)}

The expression of cancer can be divided into three categories by the scale of the disease: systemic symptoms, local symptoms, and distant metastatic symptoms.

\section{Systemic symptoms}

Systemic symptoms are symptoms that affect the whole body. Common systemic symptoms of cancer include fever, weight loss, insomnia, and hidrosis. In one study, 309 cancer survivors participating in the Rare Cancer Genetics Registry completed the Rotterdam Symptom Checklist. Patients were a median of 7.6 years post treatment. Consistent with most studies, fatigue (75\%) and insomnia (50\%) were the most commonly cited late effects [11].

\section{Local symptoms}

Local symptoms mainly occur in the area the tumor grows, such as pleural effusion caused by the presence of lung cancer. Pleural space is the place between the lung and the chest wall. The pathology of malignant pleural effusion (MPE) is the accumulation of fluid and cancer cells collected in the pleural space. When lung cancer spreads to the pleural space of the lung and increases the production of pleural fluid, as well as decreases absorption of the fluid, malignant MPE forms. Chest pain or pressure, shortness of breath at rest or with activity, and fatigue can be the outcomes of MPE [12].

\section{Distant metastasis symptoms}

Metastasis is the process of cancer, by invading normal tissues or growing into blood vessels and lymph nodes, spreading from the original emerging place to a secondary area, and forming a new tumor. Metastasis symptoms are outcomes of metastasis of malignant tumors [13]. Breast, colon, bladder, bone, and lung are common places where tumor tend to spread to. Take bone as an example. The metastasis cancer would cause pain and fracture [14].

\subsection{Factors that account for the occurrence of cancer}

\subsubsection{Chemical factors}

Exposure to chemical carcinogens can lead to an increased chance of developing cancer. These chemicals enter the body and can produce carcinogens either directly or during the metabolic process. Especially in 
large doses, the effect of carcinogens is particularly prominent. Polycyclic aromatic hydrocarbons (PAHs), a chemical constituent of cigarettes, is a common carcinogen that induces mutations in the p53 gene. It can inactivate $\mathrm{p} 53$ by generating a reactive oxidative species [15].

\subsubsection{Physical factors}

Physical factors mainly include ultraviolet radiation, magnetic radiation, and mechanical stimulation, which is also very common in daily life. For example, exposure to sunlight for too long or exposure to strong sunlight can lead to ultraviolet overdose. In cutaneous squamous cell carcinoma, UV light mutates the critical region of the gene KNSTRN, which encodes a protein that helps to form the kinetochore. Mutation in KNSTRN inhibits a cell's ability to pull apart pairs of newly replicated chromosomes to either end of the cell during cell division equally [16].

\subsubsection{Biological factors}

Viral infections, for example, have also been linked to some kinds of cancer. Many tumor formation cases are related to viral infection, such as liver cancer, nasopharyngeal cancer, breast cancer, and cervical cancer. Human papillomavirus (HPV) is a well-known virus for significantly raising the potential of cervical cancer. Persistent infection of HPV will cause normal cells in the transformation zone to mutate into cancerous cells [17].

\subsubsection{Genetical factors}

Some cancers run in families and ethnically. This means that if one person in the family has particular cancer, such as breast cancer, there is a greater chance that their direct family members will also have the same kind of cancer.

\section{Obesity and cancer}

\subsection{Obesity and risk of cancer}

As obesity has become more and more in concern in public, the association between obesity and risk of cancer is noteworthy. The risk of having cancer at up to 13 human body sites, such as the endometrium, is linked to excess body weight. Statistical analysis on indicators of obesity (which includes body mass index, waist Circumference, waist-Hip ratio, and adult-attained height) and cancer sites have revealed a significant relationship between adiposity and cancer [18].

The biological mechanisms in an increased risk of cancer caused by obesity can be grouped into three main categories: (1) Abnormalities in hormonal secretions (2) Altered microenvironment in human body (3) Different level of adipocytokines.

\subsubsection{Obesity and abnormalities in hormonal secretions}

According to previous research, obesity can induce many kinds of hormonal imbalance, such as female sex hormones (LH) and insulin. Insulin, for example, can accelerate carcinogenesis through insulin-like growth factor (IGF) mediation. The IGF system consists of two signaling molecules, IGF-I and IGF-II, six IGF-binding proteins (IGFBP-1-IGFBP-6), and several bindingprotein proteases $[19,20]$. Insulin level is altered due to insulin resistance caused by visceral and ectopic fat depots. Since insulin sensitivity reduces, insulin production increases to compensate for this change and will thus cause an elevated insulin concentration in the human body. The serum concentration of IGFBP1 and IGFBP2, attributed to hyperinsulinemia, is increased when a person is obese. As IGFBP1 and IGFBP2 repress the activity of IGF, available IGF increases [21]. IGF, after binding with receptors on the plasma membrane, can promote cell reproduction and inhibits apoptosis [22].

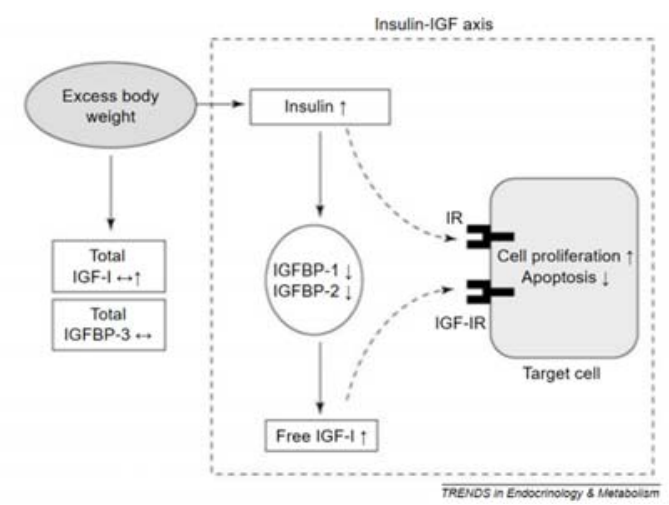

Figure 5 Association between obesity and Insulin-IGF axis

\subsubsection{Obesity and altered microenvironment in the human body}

A human small intestine is a place for the highly diverse symbiosis of microbes. Intestinal microorganisms are responsible for defensive functions and digestion. Being overweight can potentially alter the gut microbiota. Deoxycholic acid (DCA), a metabolite of gut microbes, rises in production due to the alteration effect. In turn, it promotes senescence-associated secretory phenotype (SASP) in hepatic stellate cells (HSCs). In consequence, the activity of the p53 gene is impeded by speeding up the degradation of $\mathrm{p} 53$ by the protease system. The effect of a changed microenvironment is proved to promote hepatocellular carcinoma (HCC) development in mice experiments [23].

\subsubsection{Obesity and different level of adipocytokines}

Adiponectin and leptin are two important adipokines released by adipose tissue. These 2 polypeptides crossregulate each other and have opposite relationships with obesity. Visceral and excessive ectopic fat distribution is related to decreased adiponectin concentration while associated with elevated leptin secretion. Leptin up- 
regulates the proliferation and activity of cytotoxic $\mathrm{T}$ cells while represses the action of regulator $\mathrm{T}$ cells. Adiponectin is a hormone synthesized by adipose tissue, presenting anti-inflammatory as well as insulinsensitizing properties [24]. It decreases the activity of the insulin pathway that plays a role in neoplastic progression. Thereby, when the concentration of adiponectin drops, the risk of tumor formation rises. Adiponectin level, moreover, has a negative correlation with three adipose-tissue-derived cytokines: IL-6, IL-8, and TNF-alpha [25]. These molecules can boost cancer cell growth and promote metastasis of cancer [26].

\subsection{Obesity on the treatment of cancer}

A common treatment for cancer includes surgery and radiation therapy. Previous research manifests that increased fat mass is positively correlated with recurrence and complications.

\subsubsection{Surgery}

Obesity is long-termly considered as one of the risk factors of surgery mortality. For example, both postoperational mortality in gastroenterological cancer patients and breast cancer patients are higher. Excess body fat is to blame for both the emergence and higher surgery death rate of gastroenterological cancers. Patients with BMI values larger than 30 will experience poorer short-term survival [27]. Breast-conserving surgery (BCS) or lumpectomy is an advanced way of removing the pathological breast. Obese patients, after carrying out BSC, are more likely to suffer from side effects of anesthesia due to the difficulty to maintain ventilatory support [28].

\subsubsection{Radiation therapy}

Radiation therapy (RT) is a common post-operational method to treat cancer. In this technique, ionizing radiation is directed at malignant cancer cells, killing them as well. The dose of radiation received by cancer patients is positively correlated with body mass. The study reveals that women with larger breasts in RT are almost twice as likely to face adverse changes in their breasts than medium-sized breasts [29]. Furthermore, larger BMI values (larger than or equals to 29) are also strongly associated with chronic chest pains after RT [30].

\subsubsection{Recurrence}

Not merely the treatment itself, obesity also affects aspects after the medical care - recurrence of cancer. The recurrence rate in people with large visceral fat deposits is significantly higher. In research of D. Palma, obese men had lower serum testosterone levels than overweight and normal-weight men. According to statistical analysis, their risk of biochemical recurrence of prostate cancer after RT is more prominent than the others [31]. The majority of studies of adiposity and outcomes in nonmetastatic colorectal cancer show poorer recovery in extremely obese patients (BMI larger than 35) [32].

\section{Conclusion}

As obesity is affecting more and more people in the worldwide, it is necessary to understand the mechanisms that will lead to the development and progression of specific cancers. It is becoming clear that targeting the cancer with specific therapies like using targeted medicine but it may ignore the systemic metabolic disfunction may contribute to produce resistance to those cancer therapy and treatment may be failed. As our understanding of the mechanisms and related systemic metabolism to cancer grows, it will help to take therapies with patient's conditions to specific cancer targets altered by metabolic disfunction or to identify the patients who will be benefit from those therapies to treat specific metabolic abnormalities. Also, doing exercies to loss weight can reduce the possibility of getting cancers.

\section{References}

1. NCHS 2008. Available at http://www.cdc.gov/nchs/data/hestat/overweight/ove rweight_adult.htm.

2. Calle EE, Rodriguez C, Walker-Thurmond $\mathrm{K}$ et al. Overweight, obesity, and mortality from cancer in a prospectively studied cohort of U.S. adults. N Engl J Med 2003;348:1625-1638.

3. Finucane MM, Stevens GA, Cowan MJ, Danaei G, Lin JK, Paciorek CJ, Singh GM, Gutierrez HR, Lu Y, Bahalim AN, Farzadfar F, Riley LM, Ezzati M, Global Burden of Metabolic Risk Factors of Chronic Diseases Collaborating G (2011) National, regional, and global trends in body-mass index since 1980: systematic analysis of health examination surveys and epidemiological studies with 960 country-years and 9.1 million participants. Lancet 377(9765):557567. doi:10.1016/S0140-6736(10)62037-5

4. Coates RJ, Clark WS, Eley JW et al. Race, nutritional status, and survival from breast cancer. J Natl Cancer Inst 1990;82:1684 -1692.

5. Goodwin PJ, Boyd NF. Body size and breast cancer prognosis: A critical review of the evidence. Breast Cancer Res Treat 1990;16:205-214.

6. Kroenke CH, Chen WY, Rosner B et al. Weight, weight gain, and survival after breast cancer diagnosis. J Clin Oncol 2005;23:1370-1378.

7. Fine JT, Colditz GA, Coakley EH et al. A prospective study of weight change and healthrelated quality of life in women. JAMA 1999;282:2136- 2142.

8. 7. Goodwin PJ, Ennis M, Pritchard KI et al. Fasting insulin and outcome in early-stage breast cancer: Results of a prospective cohort study. J Clin Oncol 2002;20:42-51. 
9. Reece, L.A.U.M.L.C.S.A.W.P.V.M.J.B., Campbell biology. 2017: New York, NY : Pearson Education, Inc.

10. Weinberg, R.A.J.S.A., How cancer arises. 1996. 275(3): p. 62-70.

11. Murphy, B. and J. Epstein, Chronic systemic symptoms in cancer patients, in Translational Systems Medicine and Oral Disease. 2020, Elsevier. p. 353-369.

12. Roy Semaan MD, D.F.-K.M., Christopher Slatore, MD, MS and Marianna Sockrider, MD, DrPH. Malignant Pleural Effusions. Available from: https://www.thoracic.org/patients/patientresources/resources/malignant-pleural-effusions.pdf.

13. Woodhouse, E.C., R.F. Chuaqui, and L.A.J.C.I.I.J.o.t.A.C.S. Liotta, General mechanisms of metastasis. 1997. 80(S8): p. 1529-1537.

14. Institute, N.C. Metastatic Cancer: When Cancer Spreads. 2020 November 10, 2020]; Available from: https://www.caner.gcov/types/metastatic-cancer.

15. Yu, D., et al., Reactive oxygen species generated by PAH o-quinones cause change-in-function mutations in p53. 2002. 15(6): p. 832-842.

16. Lee, C.S., et al., Recurrent point mutations in the kinetochore gene KNSTRN in cutaneous squamous cell carcinoma. 2014. 46(10): p. 1060-1062.

17. Schiffman, M., et al., Human papillomavirus and cervical cancer. 2007. 370(9590): p. 890-907.

18. Avgerinos, K.I., et al., Obesity and cancer risk: Emerging biological mechanisms and perspectives. 2019. 92: p. 121-135.

19. LeRoith, D. and C.T.J.C.1. Roberts Jr, The insulinlike growth factor system and cancer. 2003. 195(2): p. 127-137.

20. Khandwala, H.M., et al., The effects of insulin-like growth factors on tumorigenesis and neoplastic growth. 2000. 21(3): p. 215-244.

21. Renehan, A.G., et al., Obesity and cancer risk: the role of the insulin-IGF axis. 2006. 17(8): p. 328-336.

22. Moschos, S.J. and C.S.J.O. Mantzoros, The role of the IGF system in cancer: from basic to clinical studies and clinical applications. 2002. 63(4): p. 317-332.

23. Yoshimoto, S., et al., Obesity-induced gut microbial metabolite promotes liver cancer through senescence secretome. 2013. 499(7456): p. 97-101.

24. , F., C. La Rocca, and G.J.B. Matarese, Immunological functions of leptin and adiponectin. 2012. 94(10): p. 2082-2088.

25. Hebbard, L., et al., Multifaceted roles of adiponectin in cancer. 2014. 28(1): p. 59-69.

26. Kozłowski, L., et al., Concentration of interleukin-6 (IL-6), interleukin-8 (IL-8) and interleukin-10 (IL10) in blood serum of breast cancer patients. Roczniki Akademii Medycznej w Bialymstoku (1995), 2003. 48: p. 82-84.
27. Ri, M., S. Aikou, and Y. Seto, Obesity as a surgical risk factor. Annals of gastroenterological surgery, 2018. 2(1): p. 13-21.

28. Lee, K., et al., The impact of obesity on breast cancer diagnosis and treatment. Current oncology reports, 2019. 21(5): p. 1-6.

29. Moody, A., et al., The influence of breast size on late radiation effects and association with radiotherapy dose inhomogeneity. Radiotherapy and Oncology, 1994. 33(2): p. 106-112.

30. Welsh, J., et al., Obesity increases the risk of chest wall pain from thoracic stereotactic body radiation therapy. International Journal of Radiation Oncology* Biology* Physics, 2011. 81(1): p. 91-96.

31. Palma, D., et al., Obesity as a predictor of biochemical recurrence and survival after radiation therapy for prostate cancer. BJU international, 2007. 100(2): p. 315-319.

32. Demark-Wahnefried, W., et al., The role of obesity in cancer survival and recurrence. Cancer Epidemiology and Prevention Biomarkers, 2012. 21(8): p. 1244-1259. 